Article

\title{
Post-Disaster Recovery Associations of Power Systems Dependent Critical Infrastructures
}

\author{
Partha Sarker * and Henry D. Lester \\ College of Engineering, University of South Alabama, Mobile, AL 36608, USA; hlester@southalabama.edu \\ * Correspondence: pps303@jagmail.southalabama.edu; Tel.: +1-251-460-6140
}

Received: 1 April 2019; Accepted: 22 May 2019; Published: 29 May 2019

check for updates

\begin{abstract}
The complete failure of the power systems infrastructure in Puerto Rico, following Hurricanes Irma and Maria in 2017, severely hampered the recovery efforts of multiple critical infrastructure systems (CIS). Understanding the relationships of infrastructure recovery efforts between power infrastructure systems and the other CIS has the potential to be a key in developing an effective recovery plan leading to resilient infrastructure systems, and thereby a more resilient community. This paper explores the critical interfaces and interdependencies in CIS recovery by examining the disruptions and recovery progress of the CIS, including the power infrastructure systems, in Puerto immediately following the events of Hurricane Maria. This research uncovers that strong CIS recovery interdependency relationships exist between the power infrastructure systems and other CIS in Puerto Rico, and these relationships contribute to the resilience of these CIS. The resultant CIS recovery associations may potentially predict the recovery progress of post-disaster CIS recovery centered on the power infrastructure systems and lay the groundwork for further interdependency analysis of CIS in post-disaster scenarios. The results may also be helpful while designing CIS for resiliency in natural disaster areas.
\end{abstract}

Keywords: critical infrastructure systems; power systems; critical infrastructure interdependencies; post-disaster recovery; interdependency modeling; Hurricane Maria; resiliency

\section{Introduction}

Electricity and electrical energy services are essential to the proper functioning of modern-day life and power systems infrastructure fulfills this critical role of ensuring the continuous availability of the electrical energy services. Due to the continuous availability of its services, the pivotal role of power systems goes almost unnoticed in developed nations and communities. However, during powerful natural disaster events, this situation can often change dramatically with the power system infrastructure coming under severe stress that exceeds the stress handling capability of the infrastructure and its components. It is only during these disaster events, when the power system infrastructure takes severe damage, and electrical service experiences disruptions, that the paramount importance of this infrastructure system comes to the fore. As with other critical infrastructure systems (CIS) of modern communities, the power infrastructure systems has close links with other CIS and is central to the proper functioning of all the other CIS, such as transportation systems, water, natural gas and oil or petroleum, financial and telecommunication services [1]. As the society and communities evolve, these CIS are becoming more and more interlinked and interdependent on each other. Therefore, a successful investigation of power infrastructure systems and other CIS must consider the critical links and interdependencies among these CIS.

This study examines some of these critical interdependencies centered on the power infrastructure systems in the context of a major natural disaster scenario. Figure 1, as considered by Rinaldi et al. [2], shows an overview of the critical role of electrical power infrastructure systems and its external 
interdependency relationships with other CIS. The figure shows that the telecommunication system relies on power system to energize its communications switches, the transportation system relies on electrical power for its signals and switches, natural gas and petroleum infrastructures rely on electrical power to operate their storage, pumps, compression and control systems, and the water system relies on electrical power for its lift stations and control systems. Although the figure does not show, banking and financial systems are also heavily reliant on the power systems for their proper uninterrupted functioning. The figure also shows interdependencies among the CIS excluding power infrastructure systems, such as the flow of communications and cooling water between the telecommunications and water systems. However, this study does not explore these relationships as they do not involve the power infrastructure systems.

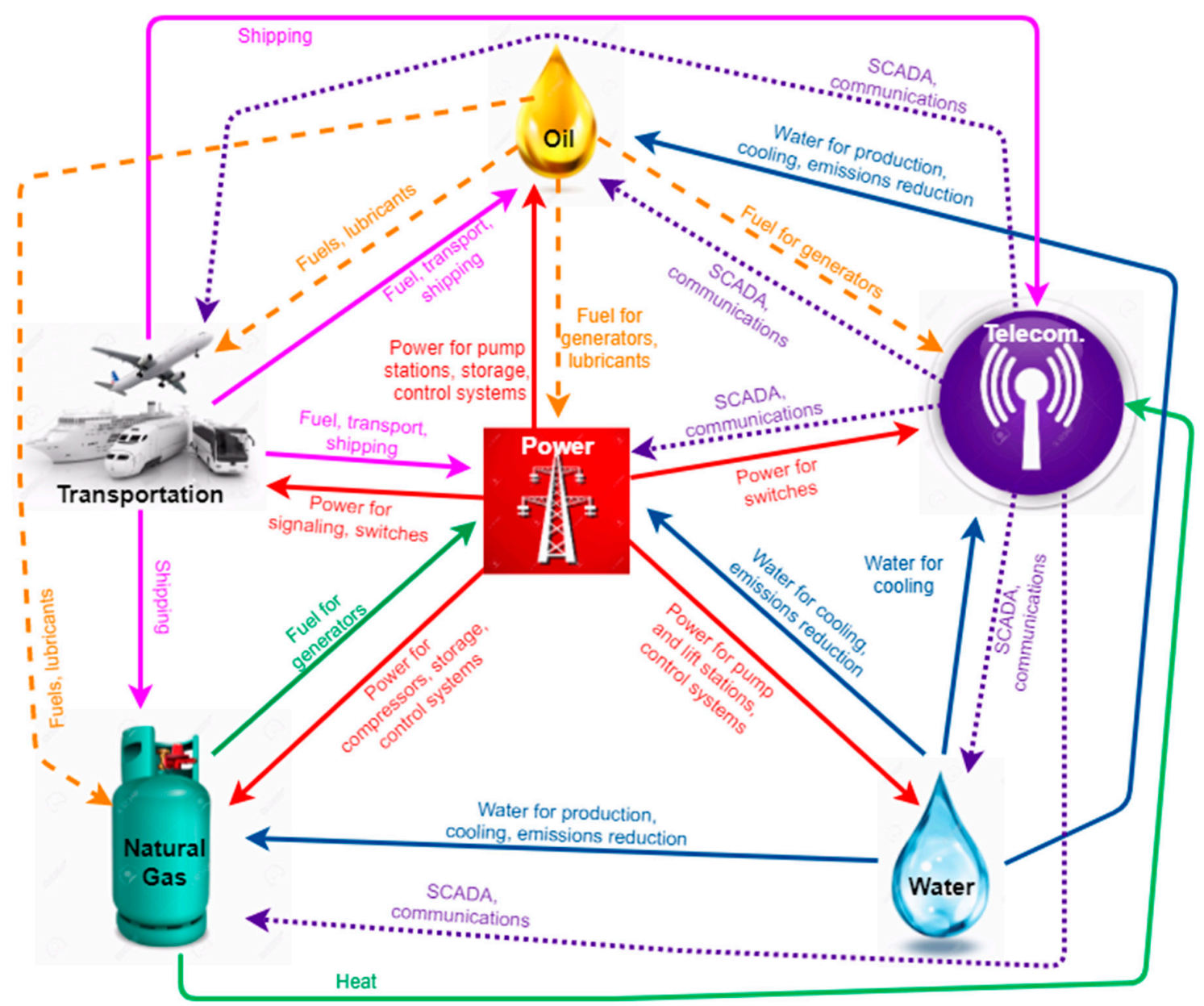

Figure 1. External interdependencies between power systems infrastructure and other critical infrastructure systems (CIS).

Understanding the dependency and interdependency relationships between power infrastructure systems and these CIS plays a key role in developing an effective recovery plan leading to a more resilient community. This study explores these CIS interdependency relations by examining Hurricane Maria induced power systems and other CIS disruptions and recovery patterns in Puerto Rico [1].

On 6 September 2017, the eye of Hurricane Irma passed just North of Puerto Rico as a category 5 hurricane, with maximum sustained winds of up to $185 \mathrm{mph}$ [3]. Two weeks later, on 20 September 2017, Hurricane Maria made landfall near Yabucoa, Puerto Rico, as a category 4 hurricane, with maximum sustained winds of up to $155 \mathrm{mph}$ [4], and passed directly over the island, wreaking havoc to its communities and infrastructures. Hurricane Irma dealt severe damage to the energy system infrastructure of the island of Puerto Rico, causing an estimated $66 \%$ customers to lose power, 
according to Puerto Rico Electric Power Authority (PREPA) [5]. Before the power systems could fully recover, Hurricane Maria made landfall and knocked out the electric grid of the entire island of Puerto Rico, with severe damages to the other critical infrastructures also. These two natural disaster events in Puerto Rico provide a unique opportunity to observe and study the interdependencies between the power infrastructure systems and other CIS, from a different perspective, namely through the observation of the CIS recovery efforts of the island.

The resultant infrastructure systems interdependencies will facilitate prediction of possible cascading failures of critical infrastructures originating from power systems failure, and as a preventive measure, aid in integrating resilience thinking into the design of power infrastructure systems, specifically in disaster-prone areas. Moreover, the findings may also be helpful in improving mitigation, response, and recovery efforts of critical energy infrastructures after a natural disaster.

The research problem that this paper explores is the study of dependencies and interdependencies between power infrastructure systems and other CIS, and identify critical interfaces and interrelations among them, leading to a resilient power infrastructure system in disaster-prone areas and communities. The research will examine the recovery and restoration patterns of the power infrastructure system and other interdependent CIS to discover the significance of recovery dependency of the CIS on the recovery of power infrastructure system. The research hopes to show that power infrastructure systems recovery is crucial to the restoration and recovery of other CIS. Therefore, to build a resilient community consisting of resilient infrastructure systems and socioeconomic state, it is essential to design a resilient energy system infrastructure, that is quick to recover, in disaster-prone areas. The bulk of the research, to explore the critical infrastructure interdependencies and recovery and restoration patterns, will involve the investigation of the complete failure of Puerto Rico's power systems following Hurricane Maria on 20 September 2017.

The research problem mentioned above raises some important questions that are necessary to investigate. These questions are: (1) What are the critical links or interdependency relationships between the power infrastructure system and other CIS in a community? (2) How to quantify the interdependencies among these infrastructure systems? (3) How are the recovery and restoration of other CIS dependent on the recovery and restoration of power infrastructure systems?

\section{Literature Review}

\subsection{Current State of Research}

\subsubsection{Defining CIS and Identifying Interdependencies}

To explore the CIS interdependencies, it is first necessary to define CIS and their interdependency relations. Critical infrastructures are technological networks, such as energy supply, transport services, water supply, oil and gas supply, banking and finance, and information and communication technology (ICT) systems [6]. These systems are critical infrastructures because they maintain essential functions of society, and the failures of these infrastructures can cause serious harm to population, economy, and national security. Critical infrastructures interact at different levels, and failure in one infrastructure may affect the functionality of other infrastructures [7]. These critical infrastructures, due to their often highly technical nature, are interdependent on each other on various levels. Critical infrastructures are dependent and interdependent, where the term dependency refers to the unidirectional relationship, and interdependency indicates a bidirectional interaction. Usually, dependencies refer to interdependencies unless otherwise specified [8]. To explore the critical roles that the CIS play in the society, and to understand the functionality of these systems, it is vital to examine their interdependencies. To this end, many researchers have explored the CIS interdependencies using various diverse methods and attempted to identify and model the critical interdependency relations. Ouyang et al. [9] identified five types of interdependencies among CIS; input dependence, mutual dependence, shared dependence, exclusive-or dependence, and co-located dependence. It is obvious from the different classifications of critical infrastructures interdependencies that there are multiple 
different ways of looking into the interdependencies, which varies according to the purpose and nature of the analyses.

\subsubsection{Risk and Vulnerability Analysis of Interdependent Infrastructures}

There is often the need for a context in identifying interdependencies among critical infrastructures. One very useful and frequently observed context is the risk and vulnerability analysis of different interdependent critical infrastructures. The literature review conducted here finds that there are several different methodological approaches and various models and simulations, to analyze risks and vulnerabilities of interdependent critical infrastructures. The vulnerabilities primarily fall into two categories: Structural vulnerability and functional vulnerability. The analysis of structural vulnerability is helpful to design or improve the infrastructures in the long term while the discussion on functional vulnerability is useful to protect them in the short term. The analysis of structural vulnerability uses only infrastructure topologies, while analysis of functional vulnerability considers operating regimes of different infrastructures [9]. Some of the reviewed articles [10,11] highlighted another important role of risk and vulnerability analysis, which is finding the critical components of the interdependent infrastructures and ranking them. Since CIS are becoming more and more interconnected with each other, failures may propagate easily between different infrastructure systems, exceeding the boundaries of a single infrastructure. Therefore, one should not study risk and vulnerability analysis in isolation [10]. In this regard, one of the reviewed articles [10] examined the interdependencies between the power system and gas pipeline infrastructures, and another article [11] examined risk and vulnerabilities of interdependent power and water systems, to identify the infrastructures' critical components. Wang et al. [11] show a common methodological framework for conducting risk and vulnerability analysis, and subsequently identifying critical components and ranking them. One can examine the framework of vulnerability and risk analysis in relation to its application for the protection and resilience of critical infrastructures by integrating different modeling perspectives that account for the complexities of the infrastructures, and considering the human factor into the analysis [12].

\subsubsection{Methods for Modeling CIS Interdependencies}

As noted earlier, there are various diverse ways to classify the interdependencies and interrelations among critical infrastructures. Moreover, the involved interdependent critical infrastructures are often complex systems with complex interfaces in-between. This section gives a brief overview of some of the unique models found in the literature study. One example of such a model [6] uses an extended cross-sector risk and vulnerability analysis (RVA) for critical infrastructures, which involves analysis of interdependencies among a set of critical infrastructures, consisting of electric power supply, water supply, transportation, and information and communication technology, using a 6-step process. The steps are: (1) Describe the initiating event; (2) identify interdependencies and perform qualitative analysis; (3) perform a semi-quantitative assessment of the risk of the scenario; (4) perform a detailed quantitative analysis of interdependencies (optional); (5) evaluate risk and measures to reduce interdependencies; and (6) cost/benefit analysis (optional) [6]. Another example model [9] proposes a methodological approach to analyze the vulnerabilities of two interdependent infrastructures comprehensively, using the steps shown in Figure 2.

Nan and Sansavini [13] presents a powerful multilayer hybrid modeling for performance assessment of critical infrastructures, only from the perspective of a single system, Swiss power system in this case, and models the interdependencies among its three critical subsystems layers; power grid, supervisory control and data acquisition (SCADA) and human operators. The article studied the power system as a combination of integrated subsystems, structured in interdependent layers; (1) systems under control; (2) operational control system; and (3) human-organizational social system [13]. 


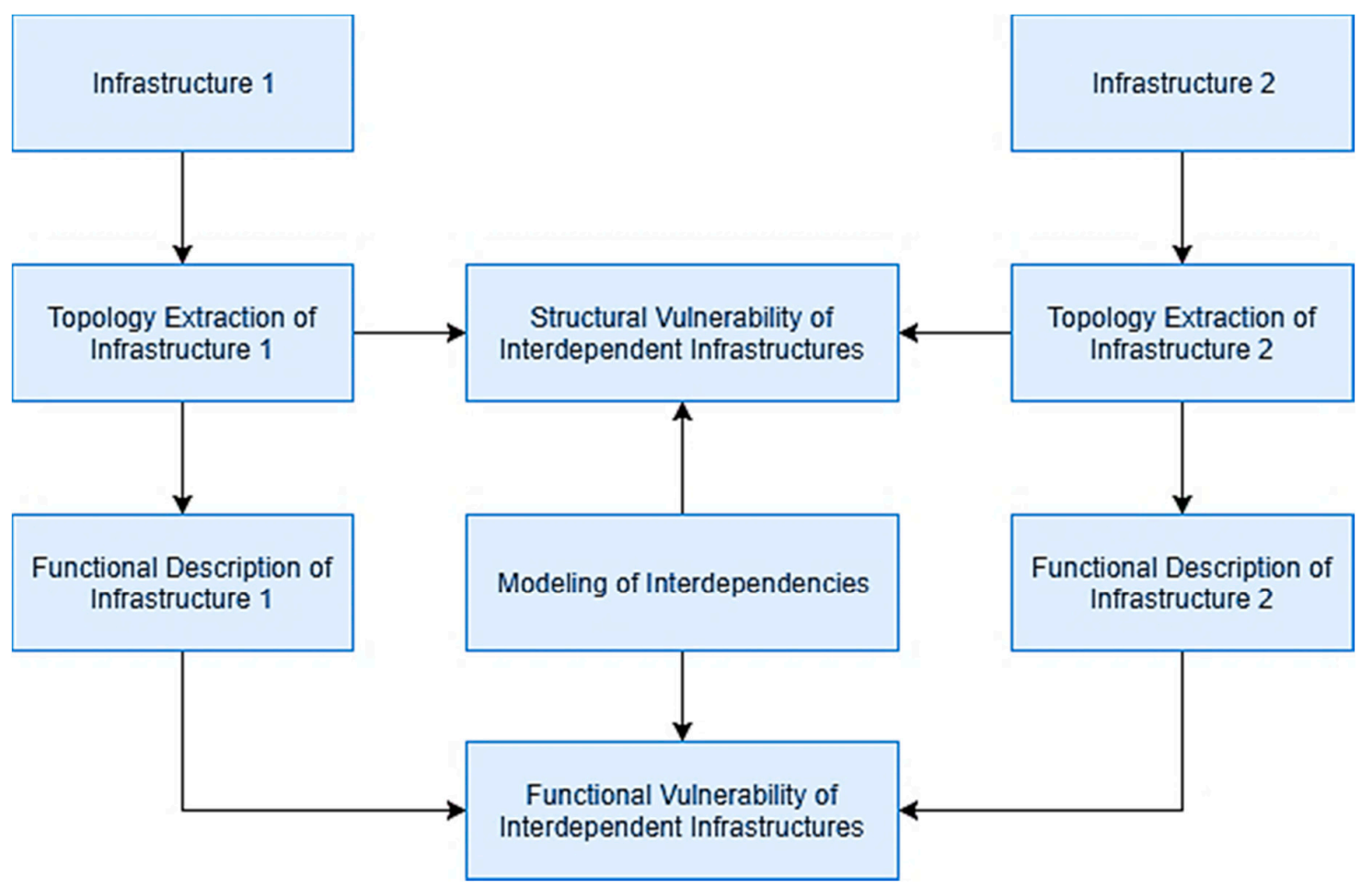

Figure 2. A methodological approach to analyze interdependent infrastructure vulnerabilities (adapted from Ouyang et al. [9]).

The literature search discovered that various researchers have used modeling approaches based on graph models or graph theory (network analysis). One such article [14], proposing a predictive modeling approach, takes a comprehensive and holistic modeling approach to study interconnected infrastructure systems. The model explores both the geographic and functional interdependencies of five interdependent systems of an electrified railway network: The railway system, traction power system, telecommunication system, auxiliary power system, and electrical in-feed system. Another journal article [15], based on graph model of interdependent networks, introduces a simulation-based method to evaluate the joint impact of interdependence, component fragilities, and cascading failures in systemic fragility estimates. This paper uses simple, directed graphs for representation of the lifeline systems or CIS, computational simulation Hazards United States Multi-Hazard (HAZUS-MH) for component fragility, and Monte Carlo simulation for systemic fragility assessment of a single distributed system [15]. Another simulation centric article [16] uses the high-level architecture (HLA) simulation standards in conjunction with agent-based modeling (ABM) using the coupled approach of the system of systems (SOS) model architecture, to represent interdependencies between two CIS, a SCADA and electric power supply. Another simplistic model [17] uses a comprehensive approach that draws on the results of a questionnaire survey of critical infrastructure experts from several countries to aid CIS operators in preparing for future crises. This paper analyzes how a failed critical infrastructure that cannot deliver products and services affects other critical infrastructures. The approach also incorporates a dynamic perspective, meaning it considers the length of time a critical infrastructure as non-operational, and follows the consequential impacts over time [17].

There are a couple of notable review articles, relevant to CIS interdependencies that provide useful insight on this topic. One is by Trucco et al. [18], which describes an integrated formalism for the dynamic functional modeling of vulnerability and interoperability of critical infrastructures at a regional level. The model assesses the propagation of impacts in terms of disservice due to a wide set of threats, and represents physical, cybernetic, geographic, as well as logical interdependencies. The model is dynamic, since both the impact of the specific threat on a generic infrastructure node and the inoperability functions are time-dependent [18]. The important finding from this article is 
the classification of different models and methodologies suggested in different papers for critical infrastructure interdependency analysis. This article provides a good summary of infrastructure systems interdependency models in existence prior to the publication of this work. According to the article, there are three levels of critical infrastructure interdependency analysis; physical, functional, and socio-economic, and there are several models used in each of these levels. The other review article by Ouyang [8] groups all the modeling approaches in use into six broad categories; empirical approaches, agent-based approaches, system-dynamics based approaches, economic theory based approaches, network-based approaches, and other approaches. The article, based on the review of over two hundred research papers and a good source of reference material on the topic of interdependency modeling, compares these six modeling approaches and suggests helpful strategies to improve the infrastructure systems resiliency.

\subsubsection{Interdependency Models Using Recovery/Restoration Data}

The literature search found some existing research works dealing with recovery and restoration of CIS, accounting for the interdependency relations among these systems. One such work [19] proposes a model based on analytical system identification methods to explore the recovery dynamics of interdependent CIS. The proposed model extracts the systems' recovery dynamics using a linear operator, called a Recovery Operator, and evaluates the value of the recovery operator using historical natural disaster recovery data to come up with an optimal recovery strategy that is computationally less intensive and expensive than more traditional high-fidelity mixed-integer programming (MIP) models. The paper recommends optimal recovery strategies in terms of restoration and recovery time of the CIS. The paper shows the implementation of the recovery operator model using a case study that involves the power, gas, and water networks of Shelby County, TN, USA, and takes into consideration their physical and geographical interdependencies.

\subsection{Research Opportunity}

The above literature review indicates that there exists a significant amount of research works involving CIS interdependencies, and many of the examples include power systems infrastructure in hypothetical case studies. However, the key finding from the review is that all the analyses, methodologies, and modeling assessments consider mostly hypothetical situations, and not necessarily a post-disaster scenario, and very few explore the recovery and restoration of these CIS in their models. There is virtually no interdependency assessment of CIS relating to power systems based on a real disaster scenario. Therefore, there is a significant research opportunity to conduct a CIS interdependency analysis centered on the power systems infrastructure by examining recovery patterns of power systems and other CIS in Puerto Rico, following the events of Hurricane Maria. As pointed out by one of the reviewed articles [20], very few studies have incorporated both the risk of power outages and the subsequent power restoration, and the research to date on power outage and restoration typically focuses on the risk analysis or the risk management, but rarely incorporates both.

\section{Research Method}

The research method proposed in the following sections tries to address the problem statement and research questions, outlined in an earlier section of this article, and fill the identified research gap. The hypothesis for the research project is that the recovery and restoration of many CIS are dependent on the recovery and restoration of power systems infrastructure. The determination of these relationships is essential to the designing of a resilient power systems infrastructure, and thereby a resilient community, in natural disaster-prone areas.

The research objectives for this project are: To identify the critical infrastructure systems that demonstrate strong interdependency relationships with the power systems infrastructure, to discover and quantify correlations of interdependent CIS with power infrastructure systems, and to study the current power infrastructure system and other CIS recovery and restoration process in Puerto Rico, 
discover the recovery pattern, and explore how they contribute to the restoration and recovery of other CIS.

\subsection{Research Data}

The data of interest for this project is the recovery and restoration data of Puerto Rico's power infrastructure systems and other CIS. Following Hurricane Maria, Puerto Rico Government set up a website [21], to track and inform the Puerto Rican public of the recovery status of these critical infrastructures. This paper uses the infrastructure recovery data from this website, and its source for telecommunications data, FCC (Federal Communications Commission) [22], and Hurricane Maria webpage of Federal Emergency Management Agency (FEMA) [23] website, to discover the interdependency relationships among the interested systems. This research involved data collection from the aforementioned sources daily and tabulation in a Microsoft Excel worksheet. Occasionally the sources did not report data daily, in which case the paper used the last reported recovery information to fill the data gaps, based on the common-sense assumption that these government sources updated the recovery data only whenever the involved parties made progress in the recovery efforts of the damaged CIS. It is worth pointing out that the power system data provided on Puerto Rico's official website [21] is the power generation data based on historical power generation information, and not the percentage of customers with electrical services. According to Ramón Rosario, secretary of public affairs, the percentage data reported reflects "the percentage we are delivering to our customers compared to what was supplied before Hurricane Maria" [24]. Therefore, the data does not reflect the total percentage of customers without power, which would be a better reflection of Puerto Rico's power system recovery. To show the recovery progress of the CIS, this paper uses the first six months or 180 days of recovery data, as by this time most of the systems reached a recovery plateau. The following results and analysis section elaborate more on this topic. To discover the recovery correlations of the CIS with the recovery of power systems infrastructure, the paper uses recovery data of the first three months or 90 days of recovery following Hurricane Maria.

\subsection{Data Analysis Method}

For the analysis of the CIS recovery data, the research used Microsoft Excel application to plot the daily recovery of these infrastructure systems. To explore the interdependency relations between power infrastructure systems and other CIS, the research used Minitab software application to plot recovery patterns of selected CIS against the recovery pattern of the power infrastructure systems and performed a linear regression analysis of the plotted data.

Linear regression is a commonly used statistical analysis method to examine relationships in bivariate quantitative data. The regression line is the line that fits a set of bivariate data points the best, by having the smallest possible sum of squared errors, i.e., the regression line meets the least-squares criterion [25]. The regression equation is the equation for the regression line, and often expressed as:

$$
\hat{y}=\beta_{0}+\beta_{1} x+\varepsilon
$$

where the coefficients are:

$$
\begin{gathered}
\beta_{1}=\frac{S_{x y}}{S_{x x}} \\
\beta_{0}=\bar{y}-\beta_{1} \bar{x}
\end{gathered}
$$

and the quantities $S_{x x}$ and $S_{x y}$ are:

$$
\begin{gathered}
S_{x x}=\sum\left(x_{i}-\bar{x}\right)^{2} \\
S_{x y}=\sum\left(x_{i}-\bar{x}\right)\left(y_{i}-\bar{y}\right)
\end{gathered}
$$


This paper uses the power systems recovery data as the predictor variable, $x_{i}$, and other CIS recovery data as the response variables, $y_{i}$, to discover the regression lines. The research analyzes the collected infrastructure recovery data as bivariate data sets, as ordered pairs of power systems recovery data $(x)$, and particular CIS recovery data $\left(y_{i}\right)$. In the above regression Equation (1), $\beta_{0}$ is the intercept of the regression line between the dependent and independent variables, $\beta_{1}$ is the slope of the regression line, and $\varepsilon$ is the error. The main purpose of this part of the research methodology is to examine whether the critical infrastructures are dependent on the power systems infrastructure, and the project examines this dependency by examining the relationships between post-disaster recovery data of critical infrastructures. The research further narrows this examination of relationships by checking for only linear relationships between the bivariate recovery data pairs. As the research method here involves a simple linear regression analysis, only bivariate data sets with linear relationships can be examined using this method. Nonlinear relationships or dependencies may exist between the data pairs, but simple linear regression analysis cannot be used to examine such an association. To check for the linear associations between a bivariate data pair, such as between the recovery data of power systems infrastructure and the recovery data of telecommunications infrastructure system, the simple linear regression checks if the regression coefficient $\beta_{1}$ (slope of regression line) is equal to " 0 " in Equation (1). If this coefficient is 0 , it indicates that there is no slope in the regression line, therefore, the telecommunications systems recovery is not associated or dependent on the recovery of power systems. If the coefficient is nonzero, it indicates a linear relationship between the recovery data pair. So, the null hypothesis to check the dependencies is:

$$
\text { Null Hypothesis }\left(\mathrm{H}_{0}\right): \beta_{1}=0
$$

and the alternative or research hypothesis is:

$$
\text { Hypothesis } 1\left(\mathrm{H}_{1}\right): \beta_{1} \neq 0
$$

Along with the regression equation, the $p$-value from the regression analysis can indicate whether to reject the null hypothesis or not. Given that the null hypothesis is rejected indicating a linear relationship, the strength of the linear relationship can be examined using the coefficient of determination or $\mathrm{R}^{2}$ values from the regression analysis, for each bivariate data pair.

\section{Results and Analysis}

\subsection{Measure of CIS Resiliency}

The daily recovery progress of the CIS reveals an important part of the existing pre-hurricane state of the resiliency of the CIS of Puerto Rico. Resilience, in the context of CIS, is the ability of the system to prepare for and adapt to changing conditions and withstand and recover rapidly from disruptions such as deliberate attacks, accidents, or naturally occurring threats or incidents [26,27]. The resilience curve in Figure 3 adapted from References $[13,28]$ illustrates the resilience of a typical critical infrastructure system that goes through a disruptive event such as a natural disaster event. The circled part of the resiliency curve indicates the part of infrastructure systems' resiliency that depends on the recoverability aspect of the CIS. The daily recovery progress of the CIS of Puerto Rico directly relates to this section of the resiliency curve, and thus reveals the pre-storm resiliency of these systems, in terms of their performance levels and hurricane preparedness. Disaster preparedness or readiness is a good way to look at these fragility curves. Although the recovery phase of resilience curve in Figure 3 shows only exponential recovery, in reality, these recovery fragility curves can be anyone of exponential, linear, of sinusoidal nature. An exponential recovery curve indicates a high level of disaster preparedness, a linear recovery indicates an average level of disaster readiness, and a sinusoidal curve indicates a low level of disaster preparedness or readiness for a given community or its critical infrastructures [26,28-30]. Following a natural disaster event, after the infrastructure 
systems pass through the recovery phase, they will attain a new normal system performance level or functionality state. This new normal functionality state for a given critical infrastructure system will follow one of three functionality levels; a permanently reduced functionality state, pre-disruption existing functionality state or an enhanced functionality state, indicated by resilience curves (a), (b), and (c) respectively in Figure 3.

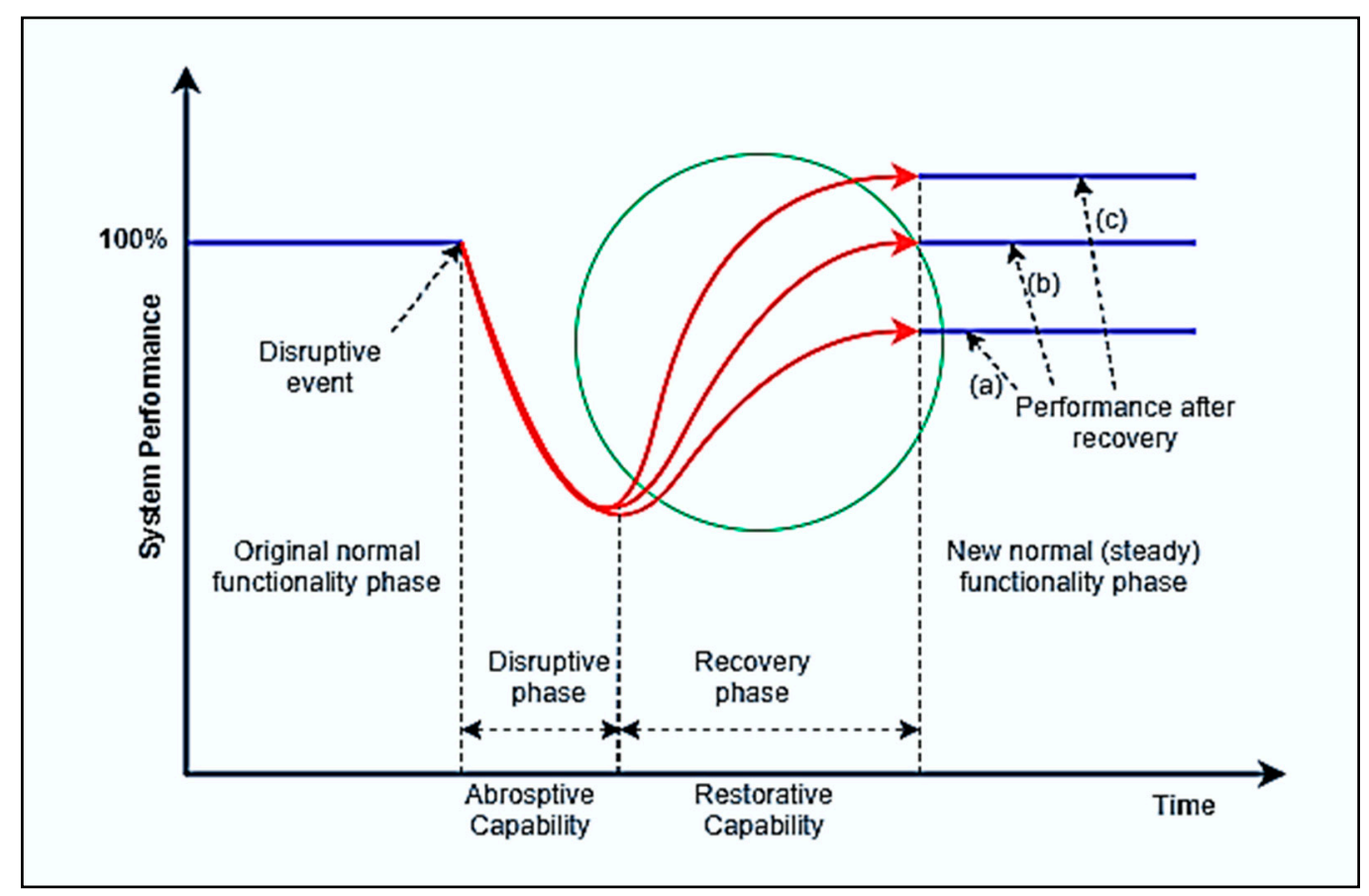

Figure 3. CIS resilience curves, in terms of systems performance or functionality (adapted from References $[13,28])$.

The recovery fragility curves in Figure 4 shows the daily recovery progress of some of the major critical infrastructures in Puerto Rico, for the first six months or 180 days (from 20 September 2017-19 March 2018), following the events of Hurricane Maria. As mentioned before, these recovery fragility curves directly relate to the Recovery Phase of the resilience curve in Figure 3. The fragility curve for the power systems infrastructure shows a very slow recovery phase. Even after a month following Hurricane Maria, the power system was only around $12 \%$ recovered, and after 3 months around $65 \%$ recovered. Even 6 months after the storm, the power systems infrastructure did not fully recover (around 95\% functionality). The recovery fragility curves also indicate the disaster preparedness or readiness of the critical infrastructures of Puerto Rico. The observation of the fragility curves for the first 90 days of recovery reveals that commercial flights, ports, AMA routes (Metropolitan bus system in San Juan), hospitals with grid electricity, banks, automatic teller machines (ATMs), supermarkets/grocery stores, gas stations, and water system infrastructures experienced exponential recovery, indicating a comparatively higher level of disaster preparedness among the critical infrastructures of Puerto Rico. The linear fragility curve of the telecommunication systems indicates an average disaster readiness, whereas the somewhat sinusoidal recovery patterns of the cell sites and power systems infrastructure indicate a comparatively lower level of disaster readiness.

The recovery curves in Figure 4 reveal some interesting observations regarding the recovery phases of some of the involved CIS. For example, the recovery of ATM systems in Puerto Rico shows a recovery well over its pre-storm system state, with a recovery of $136.02 \%$. Therefore, following the recovery phase after Hurricane Maria the ATM system has reached a new steady enhanced functionality state, following the resilience curve (c) in Figure 3. This is due to the installation of new ATMs in addition to restoring the existing ones (1586 ATMs after Hurricane Maria compared to 1166 machines 
prior to the storm). The recovery fragility curves for the supermarket/ grocery stores, gas stations, and banks, with recovered system functionality at $92 \%, 88 \%$, and $89 \%$ respectively, show a system recovery to a new steady reduced system functionality state, similar to the resilience curve (a) in Figure 3. Although this is mere speculation, these systems may never reach their pre-storm system functionality, possibly due to the relocation of several hundred thousand people from the island of Puerto Rico to the U.S. mainland [31]. However, thorough research is necessary to make accurate assertions on this issue. Figure 4 also shows that the commercial flights system recovered in the first two weeks of the disaster, and the ports reached full system functionality within the first month. For obvious reasons, these actions were necessary and a prerequisite to the undertaking of restoration of other CIS. During the early days after the storm, the responsible parties such as FEMA and U.S. Army Corp. of Engineers installed many generators to bring the flight and the port systems to full functionality. Therefore, there can be doubt about how much of these CIS were in fact fully and permanently recovered.

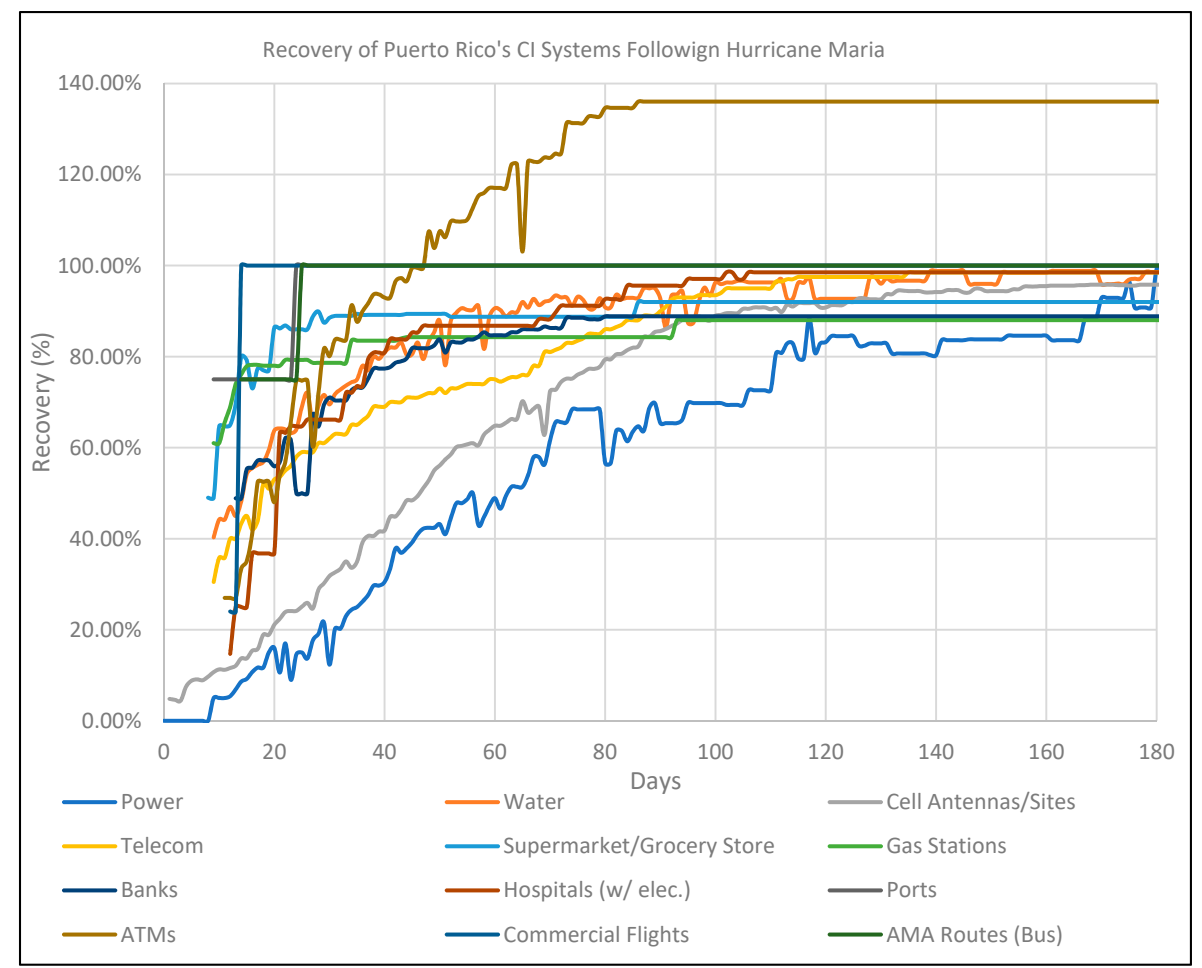

Figure 4. Daily recovery progress of CIS of Puerto Rico for the first six months after Hurricane Maria.

\subsection{Interdependency Relations between Power Systems and Other CIS}

As mentioned earlier, the article uses recovery data of the first three months or 90 days of recovery following Hurricane Maria to discover the recovery correlations of the CIS with the recovery of power systems infrastructure. Observation of the regression analysis for the bivariate data pairs reveal that $p$-values for all the data pairs are less than 0.05 , indicating that the regression equation can be used to examine the relationships between the data pairs. Consequently, observation of the regression fits and corresponding coefficient of determination or $\mathrm{R}^{2}$ values between the response critical infrastructures and the predictor power systems infrastructure reveal that the recovery of some of the critical infrastructures display relatively strong associations $\left(\mathrm{R}^{2}\right.$ value higher than $\left.50 \%\right)$ with the recovery of power systems infrastructure. These systems are; cell sites ( $R^{2}$ value $97.6 \%$ ), ATMs ( $R^{2}$ value $90.4 \%$ ), telecommunications ( $R^{2}$ value $89.3 \%$ ), water $\left(R^{2}\right.$ value $\left.85.6 \%\right)$, banks $\left(R^{2}\right.$ value $84.6 \%)$, hospitals with energized grid connection $\left(R^{2}\right.$ value $\left.71.5 \%\right)$, and gas stations ( $R^{2}$ value $53.2 \%$ ). Figure 5 graphically presents these interdependencies. The regression fits also reveal that for some CIS in Puerto Rico, the systems recovery has weak to almost nonexistent association with the recovery 
of power systems infrastructure. These infrastructure systems are; port system $\left(\mathrm{R}^{2}\right.$ value $\left.44.7 \%\right)$, AMA routes ( $R^{2}$ value $\left.37.1 \%\right)$, supermarkets or grocery stores $\left(R^{2}\right.$ value $\left.36.6 \%\right)$, and the commercial flights system $\left(R^{2}\right.$ value $\left.7.58 \%\right)$. The scatterplots with linear regression fits in Figure 6 illustrate these recovery interdependencies.

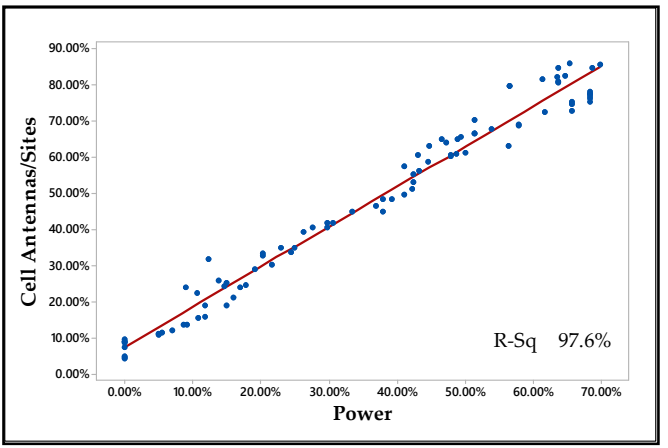

(a)

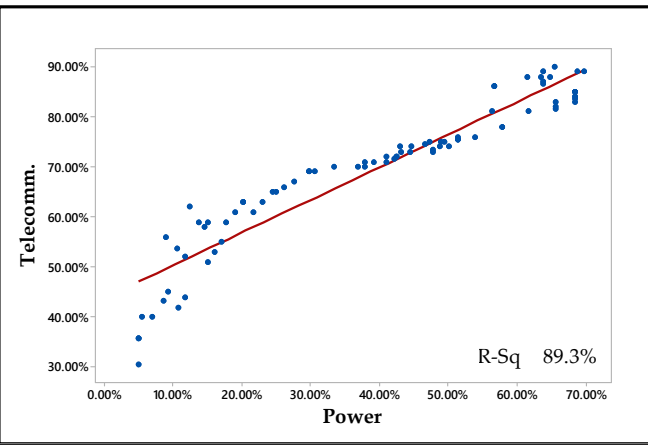

(c)

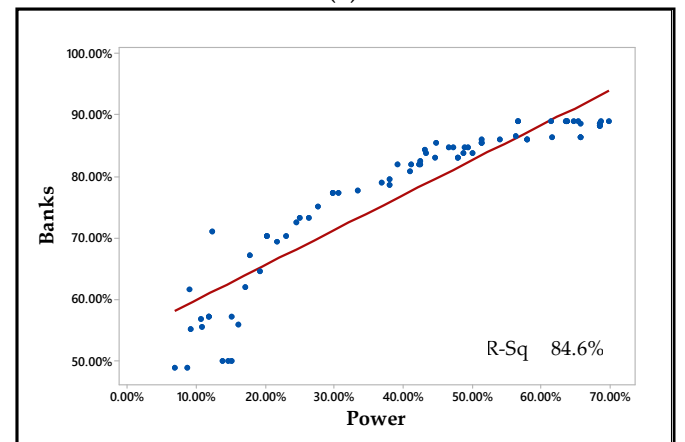

(e)

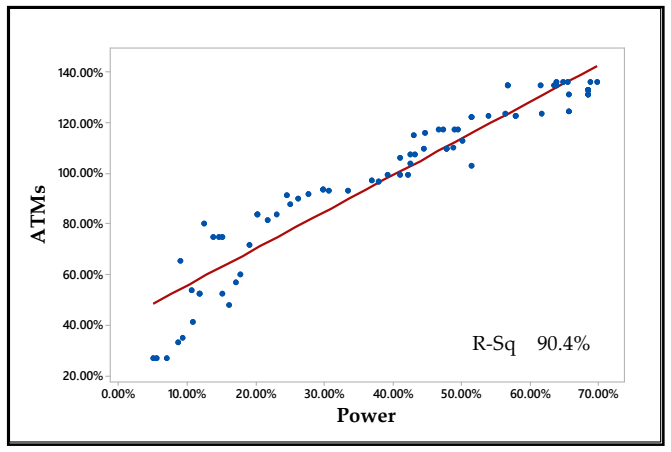

(b)

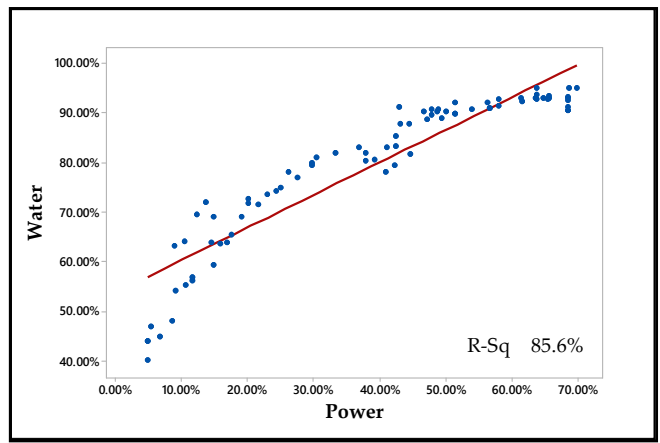

(d)

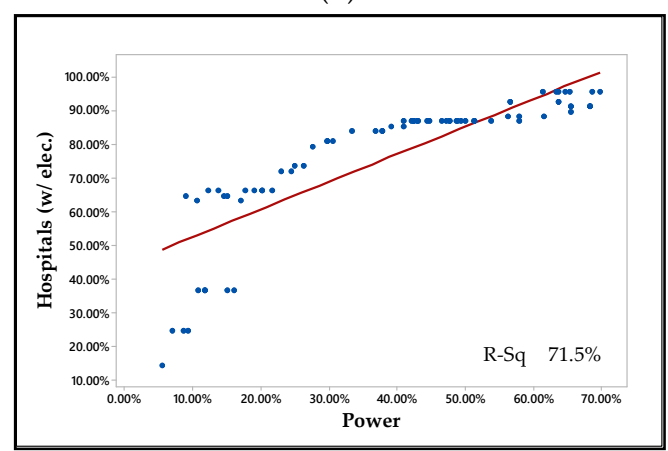

$(\mathbf{f})$

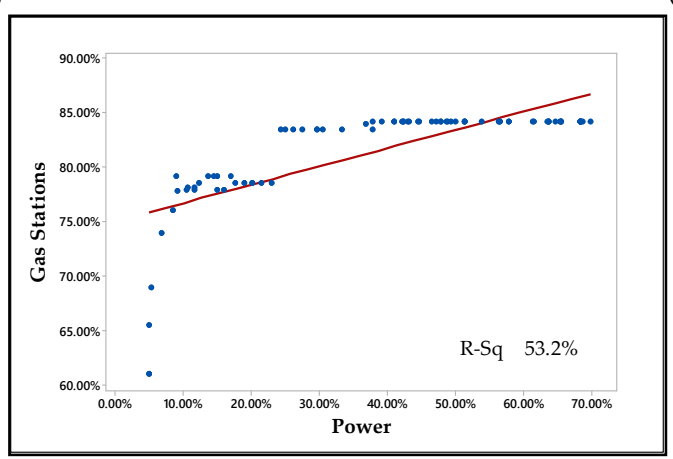

(g)

Figure 5. Infrastructure interdependency of (a) cell sites; (b) ATMs; (c) telecommunications; (d) potable water; (e) banks; (f) hospitals with grid electricity; and (g) gas station systems, with power systems infrastructure, based on post-disaster recovery patterns after Hurricane Maria. 


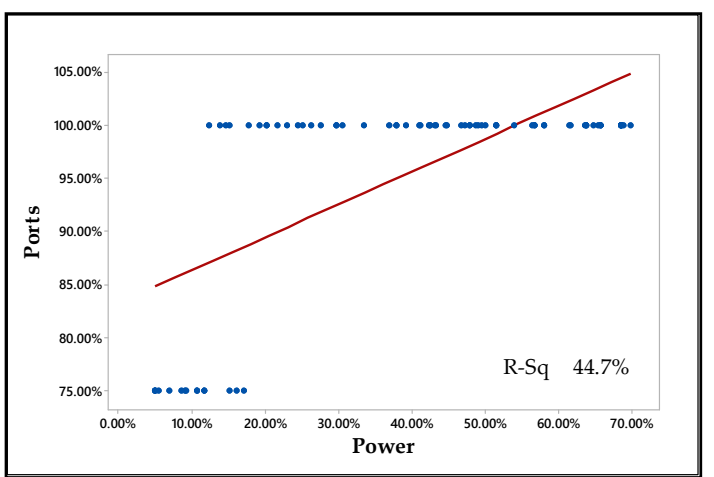

(a)

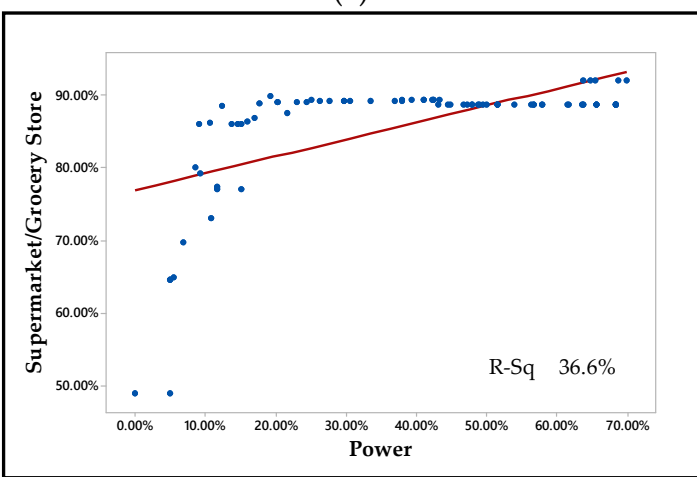

(c)

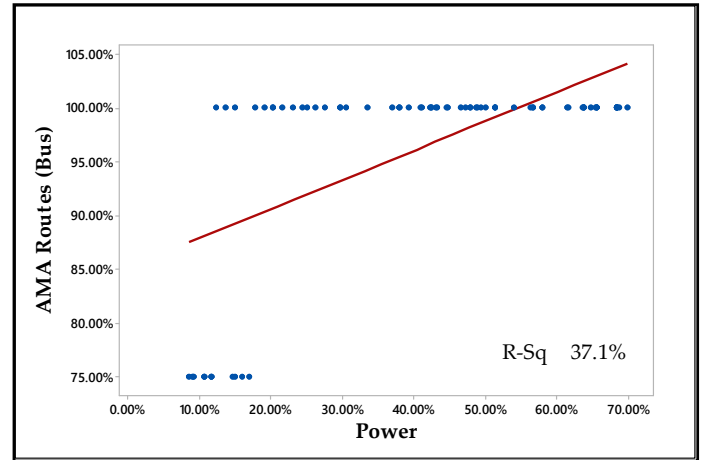

(b)

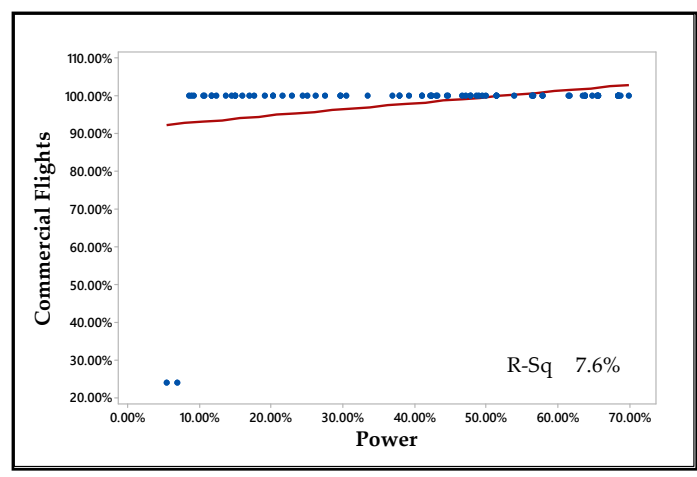

(d)

Figure 6. Infrastructure interdependency of (a) ports; (b) AMA routes; (c) supermarket/grocery stores; and (d) commercial flights systems, with power systems infrastructure, based on post-disaster recovery patterns after Hurricane Maria.

The recovery relations in Figures 5 and 6 are based on the first three months or 90 days of recovery data, following Hurricane Maria. This research considers this 90-day range for the recovery data to be a sound choice as not to skew the recovery relations between the power infrastructure systems and the other CIS. Table 1 presents a summary of the regression analyses of recovery progress interdependencies between power systems infrastructure and other CIS.

Table 1. Interdependency relations between the power system (predictor variable, $x$ ) and other CIS (response variables, $y$ ).

\begin{tabular}{cccc}
\hline Response Variable (y) & $\begin{array}{c}\text { Interdependency Relations } \\
\text { (Linear Regression Fit) }\end{array}$ & $\mathbf{R}^{\mathbf{2}}$ Value (\%) & $\mathbf{S}_{\mathbf{e}}$ Value \\
\hline Cell sites & $y=0.0756+1.1040 x$ & 97.56 & 0.0403 \\
ATM machines & $y=0.4148+1.4474 x$ & 90.43 & 0.0974 \\
Telecommunications & $y=0.4383+0.6517 x$ & 89.27 & 0.0478 \\
Potable Water & $y=0.5381+0.6547 x$ & 85.62 & 0.0567 \\
Banks & $y=0.5428+0.5670 x$ & 84.55 & 0.0488 \\
Hospitals (with grid connection) & $y=0.4448+0.8138 x$ & 71.50 & 0.1049 \\
Gas stations & $y=0.7505+0.1684 x$ & 53.21 & 0.0334 \\
Ports & $y=0.8328+0.3094 x$ & 44.66 & 0.0728 \\
AMA routes & $y=0.8517+0.2715 x$ & 37.10 & 0.0703 \\
Supermarket/grocery stores & $y=0.7693+0.2328 x$ & 36.56 & 0.0658 \\
Commercial flights & $y=0.9146+0.1631 x$ & 7.580 & 0.1163 \\
\hline
\end{tabular}

The regression equations in Table 1 depict the relationships between the power systems infrastructure and other critical infrastructure systems, and the coefficient of determination values depict the strengths of these relationships. Another important parameter from the linear regression 
analysis added in Table 1 is the standard error of the estimate, $\mathrm{S}_{\mathrm{e}}$. This standard error of the estimate parameter indicates, on average, how much the predicted values of the response variable differ from the observed values of the response variable [25]. Sometimes observations of a single regression parameter, such as the $\mathrm{R}^{2}$ values, may not show the true picture of the analysis, in which case observation of the $S_{e}$ parameter can shed more light into the results of the analysis. The extremely low values, which are desirable, for this parameter show that the regression analysis may have only minimal errors. Therefore, the results of the analysis and its parameters such as $\mathrm{R}^{2}$ values can be relied upon.

The paper focused on the linear regression analysis, as opposed to non-linear regression, for the main analysis method of the recovery data, to discover the relationships or associations that are comparatively stronger between the data pairs of the power system and other CIS. Moreover, linear regression was also used as to not force a higher $\mathrm{R}^{2}$ value between a given data pair. The purpose of choosing the linear regression here is to discover the interdependency relationships with power systems that matter most, and one way to achieve this is to discover the recovery progress that moves in unison with the recovery of the power systems infrastructure. This process also identifies the infrastructure systems that demonstrate similar levels of natural disaster preparedness as the power system. Although a non-linear regression will illustrate a more accurate nature of the relationship between the power system and any other CIS, it will not help in identifying strong interdependencies between the two systems. However, a non-linear regression will be very helpful for constructing a predictive modeling of infrastructure systems recovery, based on power systems, to predict the post-disaster recoveries of some of the critical infrastructures. Visual observation of infrastructure recovery relationships in Figure 5 point to obvious non-linear relationships for some of the infrastructures. Therefore, the paper also includes a non-linear regression analysis using the Minitab application, and Table 2 below provides a summary of the analysis. The non-linear regression analysis presented here is only to illustrate the nature of these relationships and not to draw any conclusions regarding the strength of them. The results in Table 2 only include the non-linear regression analysis for the critical infrastructures that show high non-linear relations with power system infrastructures. The infrastructures not shown on the table have either a strong linear relationship or relationships that are not appropriate for prediction of the recovery of these infrastructures based on the recovery of the power systems. The infrastructures that are not suited for a recovery prediction based on the non-linear regression analysis are ports, AMA routes, and commercial flights, most likely due to the early introduction of emergency backup temporary power sources such as generators for temporary and emergency recovery of these infrastructures.

Table 2. Important non-linear recovery relations between power system (response variable, $x$ ) and other critical infrastructure systems (response variables, $y$ ), for post-disaster recovery predictions.

\begin{tabular}{ccc}
\hline Response Variable $(\boldsymbol{y})$ & Interdependency Relation & $\mathbf{R}^{\mathbf{2}}$ Value (\%) \\
\hline Potable water & $y=1.067 x^{2}+1.477 x+0.4281$ & 94.2 \\
Hospitals (with grid connection) & $y=-9.960 x^{4}+22.47 x^{3}-18.33 x^{2}+6.762 x-0.1087$ & 90.1 \\
Supermarket/grocery stores & $y=-10.26 x^{4}+21.02 x^{3}-14.97 x^{2}+4.341 x+0.468$ & 87.5 \\
Gas stations & $y=-4.110 x^{4}+9.078 x^{3}-6.942 x^{2}+2.224 x+0.8579$ & 85.8 \\
Banks & $y=-1.032 x^{2}+1.385 x+0.4226$ & 94.3 \\
\hline
\end{tabular}

The accuracy of the interdependency relationships based on CIS recovery associations tabulated in Table 1 heavily depend on the accuracy of reported recovery data. Assuming the reported data is correct, these recovery interdependency associations with the recovery of power systems infrastructure may potentially be able to correctly predict the damage recovery patterns for some of the CIS with strong recovery associations, such as for cell sites, ATM systems, water, and banking infrastructure systems. For systems with weak recovery associations, such as for commercial flights, supermarket/grocery stores, AMA routes, and ports, the recovery pattern of the power systems infrastructure may not be the most helpful information to follow. Due to their high significance to the recovery process, CIS such as commercial flights, ports, major hospitals, and major transportation routes, are usually brought 
online using temporary power sources such as generators. Since the available recovery data does not distinguish between the availability of these systems using temporary energy sources and permanent grid-based electricity, the recovery associations presented here may not reveal the accurate state of functionality or the state of true recovery of these systems.

\section{Conclusions}

This paper presents the characteristic interdependencies between power systems and other CIS and reveals that the power system infrastructure potentially plays a key role for some CIS, in the post-disaster recovery efforts of a community, using the example of Hurricane Maria induced devastation of Puerto Rican infrastructures. The paper shows that most of the CIS, specifically cell sites, ATMs, telecommunications system, water system, banks, hospitals, and gas stations, may have strong associations in their recovery progress with the recovery progress of the power systems infrastructure. Moreover, these recovery correlations or associations may help predict the pace of recovery in a post-disaster scenario, based on the observation of power system recovery. The resulting relations also highlight the need for expedited recovery of the power systems infrastructure to ensure the speedy recovery of the strongly associated CIS.

There are certain limitations in this research work. For example, the difficulty of identifying the new steady state functionality of some of the systems that have shown either enhanced or reduced states of functionality following the recovery. Moreover, the contribution of temporary energy sources such as generators, although significant (installation of more than 703 generators in Puerto Rico and U.S. Virgin Islands, following Hurricane Maria [23]), has been difficult to identify, which can potentially skew the results of this research.

This paper presents a simple method for discovering possible linear relationships between the power system and other critical infrastructure fragility curves during disaster recovery, thereby establishing a baseline to investigate the potential interdependency influences, enhancing or impeding critical infrastructure disaster recovery. While the results of this limited study are specific to the recent hurricanes in Puerto Rico, follow-up studies should include spatiotemporal data from other extreme events and locations in conjunction with infrastructure system modeling and analysis designed to entice global conclusions regarding the underlying explanation for infrastructure recovery behavior [1]. The results here illustrate the potential associations of CIS recovery and restoration and do not make any inference regarding the underlying causation. Therefore, there is room for future work that focuses on identifying the underlying causation. Moreover, the recovery relations discovered in this paper can also provide a basis for future resilience assessments and resilience designs of power systems and other CIS, especially in a post-disaster scenario.

Author Contributions: Conceptualization, P.S. and H.D.L.; data curation, P.S. and H.D.L.; formal analysis, P.S. and H.D.L.; investigation, P.S.; methodology, P.S. and H.D.L.; project administration, P.S. and H.D.L.; resources, P.S.; supervision, H.D.L.; visualization, P.S.; writing—original draft, P.S.; writing—review \& editing, P.S. and H.D.L.

Funding: This research received no external funding.

Conflicts of Interest: The authors declare no conflict of interest.

\section{References}

1. Sarker, P.; Lester, H. Power system interruption and interdependent infrastructures disaster recovery. In Proceedings of the 2018 Industrial and Systems Engineering Conference, Orlando, FL, USA, 19-22 May 2018.

2. Rinaldi, S.M.; Peerenboom, J.P.; Kelly, T.K. Identifying, understanding, and analyzing critical infrastructure interdependencies. IEEE Control Syst. 2001, 21, 11-25. [CrossRef]

3. National Hurricane Center NOAA. Irma Graphics Archive: 3-Day Forecast Track and Watch/Warning Graphic. Available online: https://www.nhc.noaa.gov/archive/2017/IRMA_graphics.php?product=3day_ cone_with_line (accessed on 10 November 2017).

4. National Hurricane Center NOAA. Hurricane Maria. Available online: https://www.nhc.noaa.gov/archive/ 2017/al15/al152017.update.09201034.shtml? (accessed on 10 November 2017). 
5. U.S. Department of Energy-Infrastructure Security and Energy Restoration. Hurricane Irma E Hurricane Harvey Event Report (Update \#22); U.S. Department of Energy-Infrastructure Security and Energy Restoration: Washington, DC, USA, 2017.

6. Utne, I.B.; Hokstad, P.; Vatn, J. A method for risk modeling of interdependencies in critical infrastructures. Reliab. Eng. Syst. Saf. 2011, 96, 671-678. [CrossRef]

7. Setola, R.; De Porcellinis, S.; Sforna, M. Critical infrastructure dependency assessment using the input-output inoperability model. Int. J. Crit. Infrastruct. Prot. 2009, 2, 170-178. [CrossRef]

8. Ouyang, M. Review on modeling and simulation of interdependent critical infrastructure systems. Reliab. Eng. Syst. Saf. 2014, 121, 43-60. [CrossRef]

9. Ouyang, M.; Hong, L.; Mao, Z.-J.; Yu, M.-H.; Qi, F. A methodological approach to analyze vulnerability of interdependent infrastructures. Simul. Model. Pract. Theory 2009, 17, 817-828. [CrossRef]

10. Wang, S.; Hong, L.; Ouyang, M.; Zhang, J.; Chen, X. Vulnerability analysis of interdependent infrastructure systems under edge attack strategies. Saf. Sci. 2013, 51, 328-337. [CrossRef]

11. Wang, S.; Hong, L.; Chen, X. Vulnerability analysis of interdependent infrastructure systems: A methodological framework. Phys. A Stat. Mech. Its Appl. 2012, 391, 3323-3335. [CrossRef]

12. Zio, E. Challenges in the vulnerability and risk analysis of critical infrastructures. Reliab. Eng. Syst. Saf. 2016, 152, 137-150. [CrossRef]

13. Nan, C.; Sansavini, G. Multilayer hybrid modeling framework for the performance assessment of interdependent critical infrastructures. Int. J. Crit. Infrastruct. Prot. 2015, 10, 18-33. [CrossRef]

14. Johansson, J.; Hassel, H. An approach for modelling interdependent infrastructures in the context of vulnerability analysis. Reliab. Eng. Syst. Saf. 2010, 95, 1335-1344. [CrossRef]

15. Hernandez-Fajardo, I.; Dueñas-Osorio, L. Probabilistic study of cascading failures in complex interdependent lifeline systems. Reliab. Eng. Syst. Saf. 2013, 111, 260-272. [CrossRef]

16. Eusgeld, I.; Nan, C.; Dietz, S. "System-of-systems" approach for interdependent critical infrastructures. Reliab. Eng. Syst. Saf. 2011, 96, 679-686. [CrossRef]

17. Laugé, A.; Hernantes, J.; Sarriegi, J.M. Critical infrastructure dependencies: A holistic, dynamic and quantitative approach. Int. J. Crit. Infrastruct. Prot. 2015, 8, 16-23. [CrossRef]

18. Trucco, P.; Cagno, E.; De Ambroggi, M. Dynamic functional modelling of vulnerability and interoperability of critical infrastructures. Reliab. Eng. Syst. Saf. 2012, 105, 51-63. [CrossRef]

19. González, A.D.; Chapman, A.; Dueñas-Osorio, L.; Mesbahi, M.; D’Souza, R.M. Efficient infrastructure restoration strategies using the recovery operator. Comput.-Aided Civ. Infrastruct. Eng. 2017, 32, 991-1006. [CrossRef]

20. Castillo, A. Risk analysis and management in power outage and restoration: A literature survey. Electr. Power Syst. Res. 2014, 107, 9-15. [CrossRef]

21. Government of Puerto Rico. Statuspr. Available online: www.status.pr (accessed on 29 September 2017).

22. Federal Communications Commission. Communication Status Reports-Hurricane Maria. Available online: https://fcc.gov/maria (accessed on 21 September 2017).

23. Federal Emergency Management Administration. Fema Update-Hurricane Maria. Available online: https://www.fema.gov/hurricane-maria (accessed on 26 September 2017).

24. Casanova-Burgess, A.; Diaz, C. Website Gives Misleading Numbers on Puerto Rico Recovery (en Español). Available online: https://www.wnyc.org/story/puerto-rico-recovery-misleading-website/ (accessed on 10 December 2017).

25. Weiss, N.A. Introductory Statistics, 10th ed.; Prentice Hall: Upper Saddle River, MJ, USA, 2015.

26. Ayyub, B.M. Risk Analysis in Engineering and Economics, 2nd ed.; Taylor \& Francis Group: Boca Raton, FL, USA, 2014; ISBN 9781466518254.

27. The White House. Presidential Policy Directive-Critical Infrastructure Security and Resilience. Available online: https://obamawhitehouse.archives.gov/the-press-office/2013/02/12/presidential-policy-directivecritical-infrastructure-security-and-resil (accessed on 11 December 2017).

28. Lester, H.D.; Simth, R.L., III. Infrastructure System Interdependencies and Build Environment Disaster Resiliency. In Proceedings of the 2018 Industrial and Systems Engineering Conference, Orlando, FL, USA, 19-22 May 2018.

29. Ayyub, B.M. Systems resilience for multihazard environments: Definition, metrics, and valuation for decision making. Risk Anal. 2014, 34, 340-355. [CrossRef] [PubMed] 
30. Cimellaro, G.P.; Reinhorn, A.M.; Bruneau, M. Framework for analytical quantification of disaster resilience. Eng. Struct. 2010, 32, 3639-3649. [CrossRef]

31. Echenique, M.; Melgar, L. Mapping Puerto Rico's Hurricane Migration with Mobile Phone. Available online: https://www.citylab.com/environment/2018/05/watch-puerto-ricos-hurricane-migration-via-mobilephone-data/559889/ (accessed on 15 May 2018). 